\title{
Dosage de la vitamine $D$ dans le lait, par chromatographie liquide haute pression ${ }^{* *}$
}

\author{
par \\ L. MOUILLET* F. M. LUQUET* ${ }^{*}$, M. F. GAGNEPAIN* \\ et Y. SORGUE*
}

\section{INTRODUCTION}

La vitamine D est une vitamine liposoluble, formée par irradiation ultraviolette de provitamines D. Les deux représentants les plus importants sont l'ergocalciférol ou vitamine $\mathrm{D}_{2}$ (synthétisée à partir d'un précurseur végétal) et le cholécalciférol ou vitamine $\mathrm{D}_{3}$ (synthétisée à partir d'un précurseur animal).

Chez les végétaux, la vitamine $\mathrm{D}$ est peu répandue : on trouve de petites quantités de vitamine $\mathrm{D}_{2}$ dans les champignons. Dans le règne animal, on rencontre les formes $D_{2}$ et $D_{3}$ dans les huiles de foie de poissons. La vitamine $\mathrm{D}_{3}$ est aussi présente dans les tissus adipeux, les oufs et les produits laitiers renfermant de la matière grasse.

La provitamine $\mathrm{D}_{2}$ (ergostérol) se rencontre dans les levures et dans les champignons, ainsi que dans l'œuf de poule. La provitamine $\mathrm{D}_{3}$ (7 déhydrocholestérol), importante pour l'homme et les mammifères supérieurs, est concentrée dans les tissus cutanés de toutes les espèces animales, c'est à ce niveau (croit-on) qu'elle se forme.

Les vitamines $\mathrm{D}_{2}$ et $\mathrm{D}_{3}$ se dégradent assez rapidement sous l'action de l'oxygène de la lumière et des acides. En solution huileuse, il se produit une isomérisation et un équilibre s'établit entre le calciférol et le précalciférol.

L'Unité Internationale (U.I.) correspond à l'activité de $1 \mathrm{mg}$ d'une préparation standard internationale de solution huileuse de vitamine $\mathrm{D}_{3}$ ou à $0,025 \mu \mathrm{g}$ de vitamine $\mathrm{D}_{2}$ ou $\mathrm{D}_{3}$ cristallisée.

* I.S.H.A., B.P. 138, rue du Chemin-Blanc, Champlan - 91163 Longjumeau cedex. Directeur : F. M. LUQuet.

** Etude réalisée dans le cadre d'un contrat DGRST, n 7970062. 
Sur le plan des fonctions physiologiques, la vitamine D améliore la résorption du calcium à travers la paroi intestinale et facilite l'incorporation à la fois du calcium et du phosphore dans le tissu osseux. La vitamine D influence en outre l'élimination des phosphates par les reins, en augmentant leur réabsorption.

Une carence en vitamine $\mathrm{D}$ entraîne donc des troubles de la calcification de la matrice osseuse et une augmentation de l'élimination urinaire des phosphates.

L'O.M.S. situe les besoins journaliers de l'enfant vers 400 à 500 Unités Internationales. Chez l'adulte la ration nécessaire peut être portée à 1000 U.I. dans certains cas (femmes enceintes, travailleurs nocturnes).

En ce qui concerne la vitamine D dans le lait, les connaissances restent encore actuellement limitées, par manque de méthodes de dosages suffisamment rapides et précises. De plus, des enquêtes réalisées dans certains pays européens (Allemagne, Angleterre, France) ont permis de mettre en évidence quelques manifestations d'hypovitaminose $\mathrm{D}$, en particulier dans les populations urbaines.

En France, la question d'un éventuel enrichissement du lait en vitamine D a été soulevée (Conseil Supérieur d'Hygiène Publique de France, Section de l'Hygiène Alimentaire et de la Nutrition, 25-9-1979). Afin de pouvoir étudier ce problème d'enrichissement (addition ou irradiation) il est nécessaire de connaître les teneurs réelles des laits français à la production. Une telle enquête nécessite l'emploi d'une méthode d'analyse qui soit rapide et qui permette de détecter avec précision des teneurs relativement faibles en vitamine $D\left(D_{2}\right.$ et $\left.D_{3}\right)$.

\section{GENERALITES SUR LES METHODES D'ANALYSE}

Les méthodes de dosage de la vitamine $\mathrm{D}$ font appel soit à des techniques biologiques, soit à des méthodes physico-chimiques.

\section{I.1. Dosages biologiques}

On utilise le test prophylactique et curatif du rachitisme chez le rat. L'évaluation du degré de rachitisme pouvant s'effectuer en utilisant soit le "line-test " selon I'U.S.P. X V [16] soit l'examen radiologique de la calcification des os, soit le dosage gravimétrique des constituants minéraux osseux.

\subsection{Dosages physico-chimiques}

\section{I.2.1. RÉACTIONS COLORÉES}

Cette méthode a été décrite par EWIng et al. [7] et par NiELD et al. [13]. Elle consiste à développer une coloration rose en mettant 
en présence la vitamine D et le réactif de NIELD (chlorure d'antimoine dans le dichloréthane additionné de 2 p. 100 de chlorure d'acétyle. Ce complexe coloré absorbe à $500 \mathrm{~nm}$. Cette méthode doit comporter des étapes de purification sur colonne et sur couche mince afin d'éliminer certaines substances interférentes telles que la vitamine A et l'ensemble des caroténoïdes [13].

Cette méthode peut être appliquée à des échantillons dont la concentration est supérieure à 25000 U.I./g [3, 11].

\section{I.2.2. Méthodes chromatographiques}

- Chromatographie en phase gazeuse : Cette technique a été proposée par différents auteurs $[4,5,6,9,12]$. Après saponification le résidu insaponifiable peut être purifié par passages successifs sur plusieurs colonnes (celite, alumines, etc.) ou par chromatographie en couche mince. L'éluat final est repris, silylé (bis triméthylsilylacétamide), puis injecté dans le chromatographe en phase gazeuse muni d'un détecteur à ionisation de flamme. Les vitamines $D_{2}$ et $\mathrm{D}_{3}$ subissent une hisomérisation thermique, d'où l'apparition pour chacun des deux composés de deux isomères : "Pyro » et "Iso-pyro ».

Le seuil de détection de la vitamine D reste relativement élevé (400 U.I./100 g d'échantillon). Compte tenu des teneurs naturelles en vitamine $\mathrm{D}$ présentes dans les produits alimentaires, il est nécessaire de mettre en œuvre des prises d'essai importantes, d'où les nombreuses difficultés que l'on rencontre à tous les stades de la préparation.

- Chromatographie en phase liquide haute pression (C.L.H.P.) : Le développement de cette technique chromatographique a permis, dès 1972, à Williams et al. [17] de décrire une méthode de dosage des vitamines liposolubles. D'autres auteurs [1, 2, 8, 10, 14, 15] ont également étudié cette nouvelle possibilité d'analyse. Les avantages de la C.L.H.P. sont pour l'essentiel :

- de pouvoir séparer les différents isomères de la vitamine D possédant ou non des activités biologiques;

- de ne pas modifier la structure de la molécule, d'où une possibilité d'un contrôle supplémentaire à la sortie de la colonne ;

- de posséder un seuil de détection (absorption mesurée en U.V. à $254 \mathrm{~nm}$ ) suffisamment bas, permettant de doser des quantités de vitamine D de l'ordre de l'U.I./100 g d'échantillon.

L'examen des différentes possibilités de dosages nous a conduit à ne retenir, pour l'analyse des laits, que la technique par C.L.H.P. En effet, seule cette méthode est compatible avec un degré de sensibilité suffisant et une relative rapidité d'exécution permettant une enquête sur le plan national, concernant la teneur des laits crus en vitamine $D$. Nous avons conservé certains principes analytiques décrits dans de récentes publications [2, 3], tout en introduisant 
plusieurs modifications ayant pour but d'améliorer soit la précision, soit d'augmenter la rapidité de l'analyse.

\section{METHODE DE DOSAGE DE LA VITAMINE D DANS LE LAIT PAR CHROMATOGRAPHIE LIQUIDE HAUTE PRESSION}

\section{II.1. Objet}

La méthode décrite ci-après permet de doser la vitamine $\mathrm{D}_{3}$ et $\mathrm{D}_{2}$ dans le lait jusqu'à une valeur limite de 20 U.I./1.

\section{II.2. Principe}

Après saponification de l'échantillon, l'insaponifiable est d'abord extrait puis purifié, avant d'être analysé par C.L.H.P. : la détection s'effectuant en U.V. à $254 \mathrm{~nm}$.

\section{II.3. Produits et matériel}

\section{II.3.1. PRODUITS}

II.3.1.1. Ethanol absolu pour analyse.

2. Acide ascorbique.

3. Solution aqueuse d'hydroxyde de potassium à 50 p. 100 .

4. Azote R.

5. Ether de pétrole $\left(40^{\circ} \mathrm{C} / 60^{\circ} \mathrm{C}\right)$.

6. Sulfate de sodium anhydre.

7. Alumine neutre d'activité I (Woelm type 02087) désactivée à 15 p. 100 d'eau, $2 \mathrm{~h}$ avant utilisation.

8. Ether de pétrole $\left(60^{\circ} \mathrm{C} / 80^{\circ} \mathrm{C}\right)$.

9. Mélange Ether éthylique/Ether de pétrole $\left(60^{\circ} \mathrm{C}-80^{\circ} \mathrm{C}\right): 70$ : $30(\mathrm{~V} / \mathrm{V})$.

10. Digitonine (glucoside de la digitogénine). Préparer une solution éthanolique à $0,16 \mathrm{p} .100(\mathrm{P} / \mathrm{V})$.

11. Méthanol absolu pour analyse.

12. Mélange éluant HPLC : Méthanol/eau : 99,5 : 0,5 (V/V).

13. Vitamine D3 étalon.

\section{II.3.2. MATÉRIEL :}

II.3.2.1. Bain-marie à $90^{\circ} \mathrm{C}$.

2. Evaporateur rotatif (température du bain-marie $60^{\circ} \mathrm{C}$ ). 
3. Colonne de verre pour chromatographie $(\varnothing$ int : $1 \mathrm{~cm}$, longueur : $20 \mathrm{~cm}$ ), munie d'un réservoir à solvant et d'un verre fritté à sa base. Introduire l'alumine (II.3.1.7.) jusqu'à remplir la colonne sur une hauteur de $10 \mathrm{~cm}$, puis effectuer un lavage avec $50 \mathrm{ml}$ d'éther de pétrole (II.3. ).

4. Chromatograhe pour chromatographie liquide haute pression (Spectra-physics SP 8000) muni d'un détecteur par absorbance à $254 \mathrm{~nm}$ et d'un enregistreur.

5. Colonne HPLC en acier inox ( $\varnothing$ int : $0,4 \mathrm{~cm}$, longueur : $25 \mathrm{~cm}$ ). Remplie d'une phase stationnaire : lichrosorb RP $18 \mu$, (Merck).

6. Système de filtration millipore (filtre : $0,45 \mu \mathrm{m}$ ).

7. Centrifugeuse.

8. Agitateur d'ampoules à décanter (type : Agitelec).

\section{I.4. Mode opératoire}

\section{II.4.1. SAPONIFICATION :}

Introduire dans un ballon de $500 \mathrm{ml}$ :

- $100 \mathrm{ml}$ de lait;

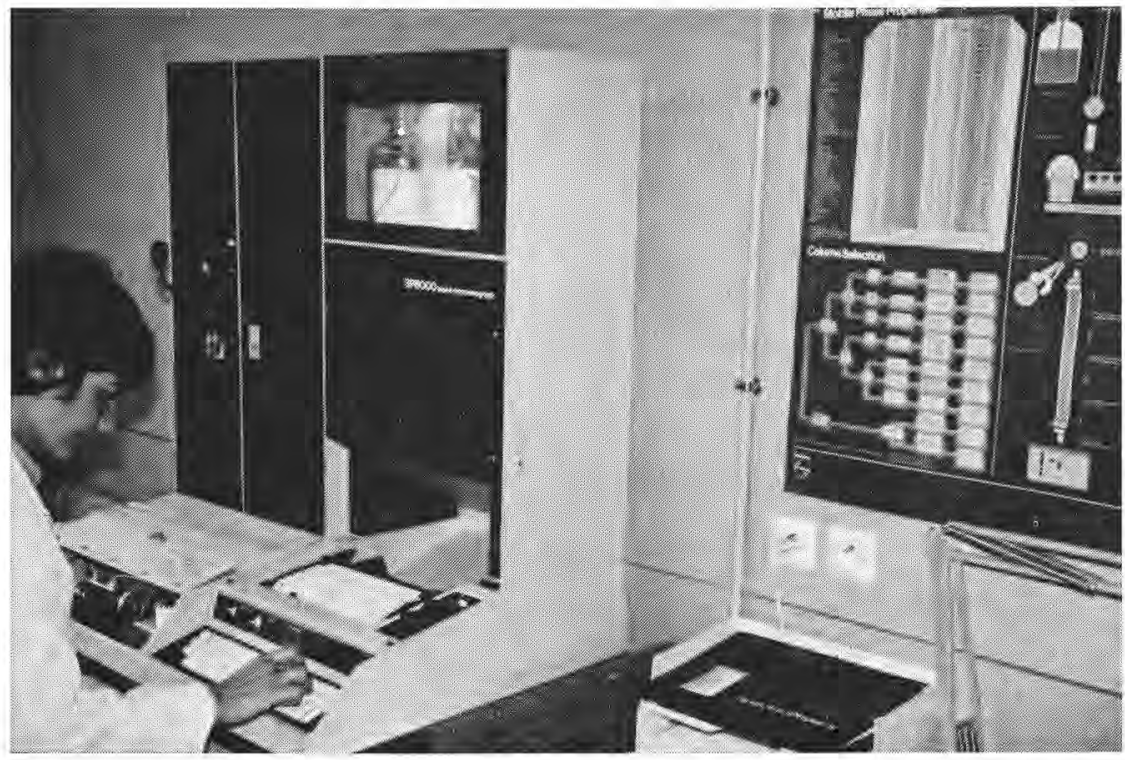

fig. 1

Chromatographie liquide SP 8000 avec intégrateur. 
- $150 \mathrm{ml}$ d'éthanol;

- 1 g d'acide ascorbique ;

- $60 \mathrm{ml}$ de la solution de $\mathrm{KOH}$ (II.3.1.3.). Mettre au bain-marie (II.3.2.1) sous réfrigérant à reflux et sous atmosphère d'azote pendant $30 \mathrm{mn}$.

\section{II.4.2 EXTRACTION DE L'INSAPONIFIABLE}

Après refroidissement, entraîner quantitativement la liqueur de saponification dans une ampoule à décanter de $500 \mathrm{ml}$ en utilisant environ $50 \mathrm{ml}$ d'eau distillée. Ajouter environ $100 \mathrm{ml}$ d'éther de pétrole (II.3.1.5.) et agiter (II.3.2.8.) pendant $2 \mathrm{~min}$.

Recommencer deux fois l'extraction en utilisant à chaque fois environ $100 \mathrm{ml}$ d'éther de pétrole. Réunir les extraits renfermant l'insaponifiable.

Laver la phase éthérée avec de l'eau jusqu'à neutralité à la phénolphtaléine, et éliminer les traces d'eau en effectuant une filtration sur du sulfate de sodium (II.3.1.6.).

Eliminer complètement le solvant à l'aide d'un évaporateur rotatif (II.3.2.2.).

\section{II.4.3. PuRification DE L'EXTRAit inSAPONIFIABLE}

\section{- Chromatographie sur alumine}

Reprendre le résidu avec $10 \mathrm{ml}$ d'éther de pétrole (II.3.1.8.), l'introduire dans la colonne (II.3.2.3.). Rincer avec $50 \mathrm{ml}$ d'éther de pétrole (II.3.1.8.), ce qui permet d'éliminer la plupart des caroténoïdes. Effectuer l'élution des vitamines liposolubles à l'aide de $70 \mathrm{ml}$ du mélange Ether éthylique/Ether de pétrole (II.3.1.9.). Evaporer à sec l'éluat.

\section{- Précipitation d'une partie des stérols par la digitonine}

Les stérols forment avec la digitonine des composés d'addition équimoléculaires peu solubles dans l'éthanol. Cette propriété est utilisée pour éliminer une fraction importante des stérols, ce qui permet d'obtenir des chromatogrammes plus facilement exploitables, Reprendre le résidu sec avec $5 \mathrm{ml}$ de la solution alcoolique de digitonine (II.3.1.10). Mettre au froid à $-18^{\circ} \mathrm{C}$ pendant $15 \mathrm{~min}$. Evaporer à $\mathrm{sec}$ et reprendre le résidu avec 5 à $10 \mathrm{ml}$ de méthanol. Centrifuger 5 min à 3000 tours min, et recueillir le surnageant. Laver le culot de centrifugation avec quelques $\mathrm{ml}$ d'éther de pétrole et effectuer une deuxième centrifugation. Eliminer le culot de centrifugation, réunir les phases éthérées et évaporer à sec.

- Filtration sur membrane millipore (II.3.2.6.)

Reprendre le résidu sec avec environ $1 \mathrm{ml}$ de méthanol. Filtrer sur membrane millipore en effectuant plusieurs rinçages en utilisant 
des fractions de $1 \mathrm{ml}$ de méthanol. Evaporer à sec et ajuster exactement à $1 \mathrm{ml}$ avec du méthanol.

\section{Remarques}

1. Toutes les manipulations doivent être faites à l'abri de la lumière directe.

2. Une purification supplémentaire peut être réalisée par chromatographie sur Célite/Magnésie telle qu'elle a été décrite par ANTALICK J.P. et al. [3]. Toutefois dans le cas du lait, cette purification n'est pas nécessaire.

\section{II.4.4. DoSAGE}

L'analyse par CLHP de l'extrait purifié est réalisée dans les conditions suivantes :

- volume injecté : $50 \mu 1$;

- colonne à polarité de phase inversée (II.3.2.5.) ; $\min$;

— phase mobile : mélange méthanol/eau (II.3.1.12.) débit : $1 \mathrm{ml} /$

- détection U.V. à $254 \mathrm{~nm}$.

Dans ces conditions, le profil chromatographique obtenu est semblable à celui représenté par la figure 2 .

Effectuer sur 1 échantillon un ajout de vitamine $\mathrm{D}_{3}$ avant saponification, ce qui permet à la fois d'identifier et de quantifier le pic de vitamine $\mathrm{D}_{3}$.

Calculer la surface des pics, soit à l'aide d'un intégrateur, soit en effectuant le produit de la hauteur par la largeur à mi-hauteur. La teneur en vitamine $D_{3}$ (U.I./1) est donnée par la formule :

$$
\text { U.I. } / 1=\frac{\mathrm{s}}{(\mathrm{S}-\mathrm{s})} \cdot \text { A. } 10 .
$$

$\mathrm{A}=$ quantité de vitamine $\mathrm{D}_{3}$ ajoutée (U.I.) dans $100 \mathrm{ml}$ de lait.

$\mathrm{s}=$ surface du pic sans ajout.

$S=$ surface du pic avec ajout.

Le calcul peut aussi être effectué à partir d'un étalonnage externe à condition de connaître le taux de perte en vitamine $\mathrm{D}_{3}$ occasionné par les différentes étapes de l'extraction et de la purification. Dans les conditions opératoires décrites ci-dessus, nous avons établi que le taux de récupération de la vitamine $\mathrm{D}_{3}$ est de l'ordre de 80 p. 100 . 

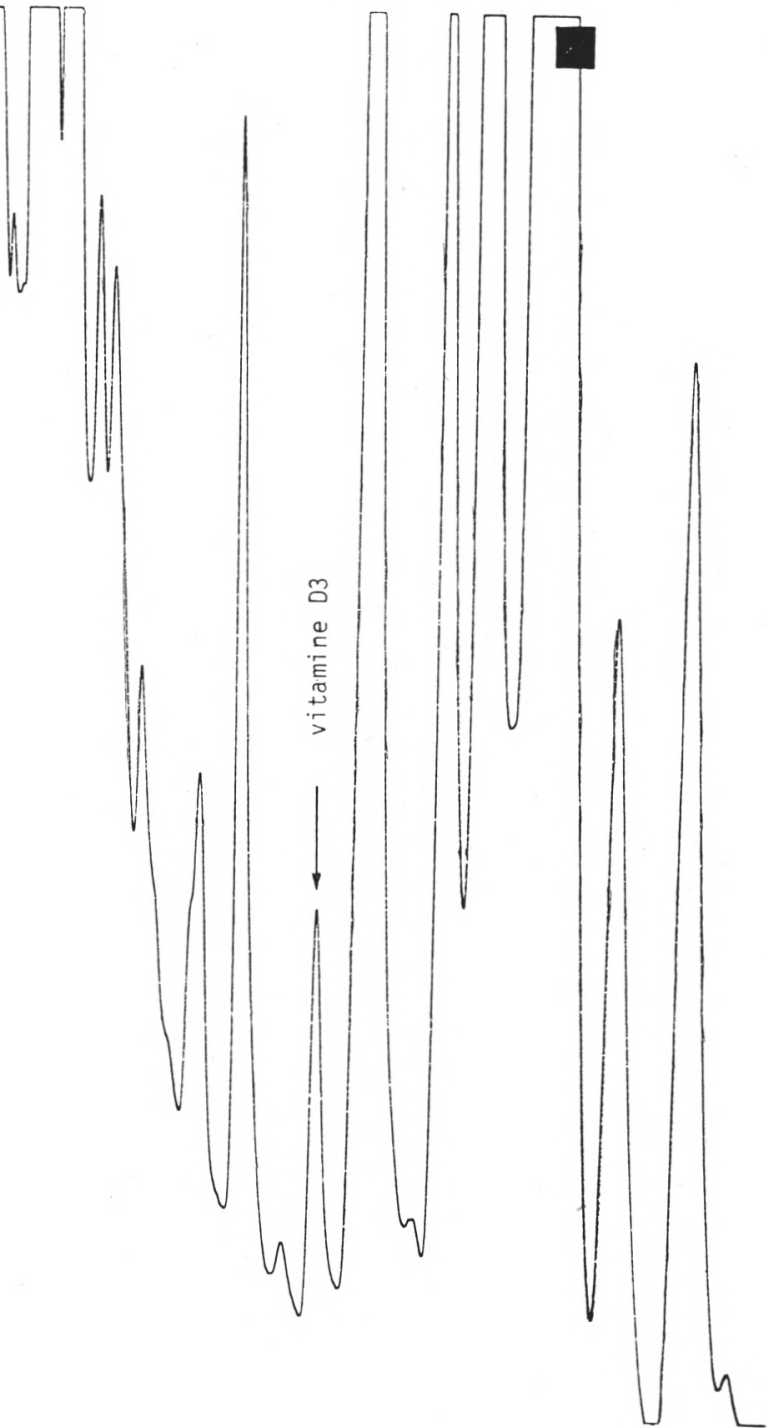

fig. 2

CLHP (profil chromatographique d'un lait cru (conditions analytiques : Cf. II-4-4). 


\section{TENEURS EN VITAMINE D3 DE QUELQUES LAITS FRANÇAIS}

Des analyses ont été réalisées sur des échantillons de laits crus de mélange, provenant de différentes régions françaises. Les résultats indiqués représentent la moyenne de plusieurs déterminations, effectuées sur des échantillons différents qui, pour une même région, ont été prélevés le même jour. Tous les prélèvements ont eu lieu durant les mois de septembre et octobre 1980.

- Région Nord : 29 U.I./1 (M.G. : 40,25 g/1).

- Région Normande :

a) 24 U.I./1 (M.G. : 41,0 g/1).

b) 25 U.I./1 (M.G. : 42,0 g/1).

c) 55 U.I./1 (M.G. : $44,0 \mathrm{~g} / \mathrm{l}$ ).

- Région Bretagne : 36 U.I./1 (M.G. : 41 g/1).

- Région Ile-de-France :

a) 40 U.I./1 (M.G. : $39,0 \mathrm{~g} / \mathrm{l}$ ).

b) 34 U.I./1 (M.G. : 38,5 g/1).

- Région Nord-Alpes : 25 U.I./1 (M.G. : 40,5 g/1).

- Région Yonne :

a) 50 U.I./1 (M.G. : 43,0 g/l).

b) 30 U.I./1 (M.G. : 40,5 g/1).

\section{CONCLUSION}

La recherche et le dosage de la vitamine $\mathrm{D}_{3}$ dans le lait nécessitent la mise en œuvre de techniques analytiques très sensibles. La chromatographie liquide haute pression permet d'être à la fois suffisamment sensible, reproductible et relativement rapide pour pouvoir être utilisée comme méthode de routine. La réalisation d'une pré-enquête sur la teneur en vitamine D de laits français a permis de constater que selon l'origine des échantillons, la vitamine $\mathrm{D}_{3}$ est présente dans les laits crus, dans des limites variant de 25 à 50 U.I./1. Il serait intéressant de pouvoir affiner ces chiffres en étudiant notamment l'influence de certains paramètres zootechniques (races, alimentation, etc.) ainsi que l'évolution au cours de l'année. Enfin, l'étude de la vitamine $\mathrm{D}$ reste encore incomplète tant que l'on n'a pas à sa disposition une méthode de dosage suffisamment précise de l'ester sulfate de cholécalciférol et de l'hydro 25 cholécalciférol. 


\section{Remerciements}

Nous remercions M. le professeur Raoul (professeur Faculté de Pharmacie, Paris), M. le professeur Pellerin (professeur Faculté de Pharmacie, ChâtenayMalabry), Mlle Le Boulch (I.N.S.E.R.M.) qui, grâce à leurs conseils et leurs encouragements ont contribué à la réalisation de cette étude.

\section{Rés u mé}

Après saponification et extraction de l'insaponifiable, la vitamine $\mathrm{D}_{3}$ est dosée dans le lait par chromatographie liquide haute pression. La technique proposée permet le dosage de la vitamine D jusqu'à une teneur limite de 20 U.I./1. Une pré-enquête réalisée en automne 1980 a montré que les teneurs en vitamine $\mathrm{D}_{3}$ des différents laits français analysés varient de 25 à 50 U.I./1.

\section{Su m m a ry}

\section{DETERMINATION OF VITAMIN D IN MILK BY HPLC}

After saponification and extraction of unsaponifiable matter, vitamin $\mathrm{D}_{3}$ is determined in milk by high pressure liquid chromatography. The technique we propose allows to proportion vitamin D up to a limited content of 20 U.I. a liter. Preliminary investigations realised during autumn 80 proved that vitamin $D_{3}$ contents, in various analysed french milks, differ from 25 to 50 U.I. a liter.

Reçu pour publication en juillet 1981.

\section{Bibliographie}

[1] Allen (C.), Ray (Y. J.), Nelson Dwyer and Reager (J. C.) (1977). - High Pressure Liquid Chromatographic Determination of Vitamine $D_{3}$ in Livestock Freed Supplements. J.A.O.A.C., 60, (6), 1296-1301.

[2] Antalick (J. P.) (1979). - Dosage des vitamines A, D, E par chromatographie liquide haute pression. Ann. Fals. Exp. Chim., 72, (780), 571-575.

[3] Antalick (J. L.), Debruyne (H.) et Faugère (J. G.) (1977). — Le dosage de la vitamine $\mathrm{D}$ dans les aliments par chromatographie liquide, haute pression. Ann. Fals. Exp. Chim., 70, (757), 497-502.

[4] Bell (G.) and Christie (A. A.) (1973). - Gas liquid chromatographic determination of vitamine D in cod liver oil. Analyst, 98, 268-273.

[5] Bellomonte (G.). - Gaz chromatographic determination of vitamine D in Premixe. Commission Experts C.E.E. Cité par AnTALICK J. L. et al. [3].

[6] BRendel (R.) und Janecke (H.) (1972). - Zur Bestimmung kleinster Mengen Vitamin D in biologishem Material. I: Gas-flüssig-chromatographie der vitamin D. Z. Anal. Chem., 258, 114-117. 
[7] Ewing (E. T.), Kingsley (G. V.), Brown (R. A.) and Emmet (A. D.) (1943). Determination of vitamins D in fish liver oil. Ang. Eng. Chem. Anal., $15,301$.

[8] Hofsass (H.), Grant (A.), Alicino (N. J.) and Greenbaum (S. B.) (1976). High pressure liquid chromatographic détermination of vitamin $D_{3}$ in resins, oils, dry concentrates, and dry concentrates containing vitamin A. J.A.O.A.C., 59, (2), 251-260.

[9] Le Boulch (N.), Gulat-Marnay (C.) et Raoul (Y.) (1974). - Dérivés de la vitamine $D_{3}$ des laits de femme et de vache : ester sulfate de cholécalciférol et hydroxy. 25 cholécalciférol. Internat. J. Vit. Nutr. Res., 44, (2), 167-179.

[10] Modest Osadca and Mercedes Aranjo (1977). - High Pressure Liquid chromatographic separation and identification of vitamins $\mathrm{D}_{2}$ and $\mathrm{D}_{3}$ in the presence of fat soluble vitamins in dosage forms. J.A.O.A.C., 60, (5), 993-997.

[11] Mulder (F. J.), DE Vries (E. J.) and Borsje (B.) (1971). - Analysis of fat soluble vitamins. J.A.O.A.C., 54, (5), 1168-1164.

[12] NAIR (P. P.) and Stella DE Leon (1967). - Current research on gas liquid chromatography of the vitamin D group. Progr. Biochim. Pharmacol., 3, 498-505.

[13] Nield (C. H.), Russel (W. C.) and Zimmerli (A.) (1940). - A. Spectrophotometric determination of vitamins $\mathrm{D}_{2}$ and $\mathrm{D}_{3} . J$. Biol. Chem., 136, 73.

[14] Pellerin (F.) et Dumitrescu (1979). - Dosage des vitamines lipo et hydrosolubles dans les préparations polyvitaminées par chromatographie liquide haute performance. Talapta., 27, 243-251.

[15] Ruckemann (H.) et RanfFt (K.) (1977). - Méthodes pour doser les vitamines au moyen de la chromatographie liquide haute performance. Zeitschrift für Lebensmittel., 164, (4), 272-273.

[16] Vitamine Compendium (1970). - Hoffmann (F.) et La Roche (A. G.), Basel, Schweiz.

[17] Williams (R. C.), Schmidt (J. A.) and Henry (R. A.) (1972). - Quantitative Analysis of fat soluble vitamins by high speed liquid chromatography. J. Chrom. Sci., 8, 494-501. 